\title{
Impact of chronic kidney disease on in-hospital mortality and clinical outcomes of catheter ablation of ventricular tachycardia: Insights from the national readmission database
}

\author{
Mahmoud Khalil ${ }^{1}$, Ahmed Maraey ${ }^{2}$, Amro Aglan ${ }^{3}$, Emmanuel akintoye ${ }^{4}$, Mahmoud \\ Salem $^{5}$, Ahmed Elzanaty ${ }^{6}$, Ahmed Younes ${ }^{7}$, Ayman Saeyeldin ${ }^{8}$, Chirag Barbhaiaya ${ }^{9}$, and \\ Mohamed Shokr ${ }^{9}$ \\ ${ }^{1}$ Lincoln Medical and Mental Health Center \\ ${ }^{2}$ North Dakota University System \\ ${ }^{3}$ Lahey Hospital and Medical Center \\ ${ }^{4}$ Cleveland Clinic Foundation \\ ${ }^{5}$ Baylor Health Care System \\ ${ }^{6}$ University of Toledo College of Medicine \\ ${ }^{7}$ East Carolina University \\ ${ }^{8}$ Saint Mary's Hospital Finkelstein Health Sciences Library \\ ${ }^{9}$ New York University Medical Center
}

November 30, 2021

\begin{abstract}
Background: Catheter ablation is an effective treatment for ventricular tachycardia (VT), albeit the decision to undergo this procedure is often influenced by underlying comorbidities. The present study aims at evaluating the effects of chronic kidney disease (CKD) on clinical outcomes of VT ablation. Methods: We identified 7,212 patients who presented between 20162018 and underwent catheter ablation for VT. Their clinical data were retrospectively accrued from the national readmission database (NRD) using the corresponding diagnosis codes. We compared clinical outcomes between patients with chronic kidney disease (CKD-group) and patients without. Odds ratios (OR) for the primary and secondary outcomes were calculated, and multivariable regression analysis was utilized to adjust for confounding variables. Results: Compared with patients without CKD, patients in CKD-group were older (mean age 67.9 vs. 60.5 years, $\mathrm{P}<0.01$ ), had a longer mean length of stay (8.73 vs. 5.69 days, $\mathrm{P}<0.01$ ), and higher in-hospital mortality (OR 2.24, 95\% confidence interval (CI) $(1.29-3.88), \mathrm{P}<0.01)$. CKDgroup patients had increased risk of developing acute kidney injury $(10 \%$ vs. $8.6 \%, \mathrm{P}<0.01)$ and 30 -day readmission rate due to VT (OR 1.77, 95\% CI (1.17-2.69), P<0.01). Conclusion: In patients with CKD, VT ablation is associated with worse clinical outcomes in-hospital mortality and 30-day readmission rate. This significantly influences the decision-making prior to performing this procedure.
\end{abstract}

\section{Introduction:}

Ventricular tachycardia (VT) commonly develops in patients with structural heart disease, especially those with myocardial scar, such as prior myocardial infarction. [1,2] Catheter ablation has been found to reduce incidence of VT storm, cardiovascular disease related hospitalization, and implanted cardioverter defibrillator (ICD) shocks when compared to medical therapy alone in patients with ischemic cardiomyopathy and structural heart disease. $[3,4]$ 
Adverse events related to VT ablation include tamponade, stroke, and acute myocardial infarction. [5] chronic kidney disease (CKD) is associated with an increased incidence of adverse events in cardiovascular procedures such as percutaneous coronary interventions [6]. The aim of our study is to evaluate the impact of CKD on in-hospital mortality and clinical outcomes of VT ablation.

\section{Methods:}

\section{Data source}

This is a retrospective cohort study using the Agency for Healthcare Research and Quality's Healthcare Cost and Utilization Project (HCUP) Nationwide Readmission Database (NRD) for the years 2016-2018. The NRD is a large, publicly available all-payer inpatient health care readmission database in the United States. The NRD is drawn from HCUP State Inpatient Databases (SID) containing verified patient linkage numbers that can be used to track a person across hospitals within a State, while adhering to strict privacy guidelines. National estimates were produced using sampling weights provided by the sponsor. All values presented are weighted estimates.

\section{Study population:}

Our study population was patients with a primary diagnosis of ventricular tachycardia who underwent catheter ablation. ICD 10 codes (025K3ZZ, 025L3ZZ, 025M3ZZ, 02583ZZ). Patients were excluded if they were younger than 18, had a primary insurance other than Medicare, Medicaid, private insurance, or selfpay. We excluded patients having a secondary diagnosis of paroxysmal supraventricular tachycardia, atrial fibrillation, atrial flutter, ventricular premature beats, pre-excitation syndrome, AV nodal tachycardia, and atrial tachycardia. Also, to avoid inclusions of patients undergoing ablation of the AV junction, we excluded patients with diagnostic or procedural codes indicating prior or current implantation of a pacemaker. December discharges and patients who died in index admission were excluded on evaluating 30 days readmission outcomes. Patient characteristics included age, gender, median household income and primary insurance. Hospital characteristics included hospital bed size and teaching status.

In accordance with the HCUP data use agreement, we excluded any variable containing a small number of observations ([?]10) that could pose risk of person identification or data privacy violation. This approach for identification of admissions with AF and catheter ablation has been previously described and used by other investigators [3]

\section{Study outcomes:}

Primary outcome was in-hospital mortality. This variable was directly coded in NRD.

Secondary outcomes were length of stay, total charges, acute kidney injury, iatrogenic cardiac complication, post procedure bleeding, cardiac tamponade, hemopericardium, stroke, acute myocardial infarction, and readmission with a primary diagnosis of ventricular tachycardia within 30 days. Length of stay and total hospital charges were directly coded in NRD. Index admission was defined as the first admission with the primary diagnosis of ventricular tachycardia without prior admission in 30 days period. A readmission was defined as any readmission within 30 days of the index admission.

\section{Statistical analysis:}

Data Analysis was performed using STATA 17 (STATA CORP College Station, Texas). Continuous variables were compared using Student t-test and categorical variables were compared using Chi-square test. Univariate regression analysis was used to calculate unadjusted odds ratio (OR) for the primary and secondary outcomes. A multivariate regression analysis was used to adjust for confounders; age, sex, median income, primary insurance, hospital bed size, hospital teaching status and chronic comorbidities such as Hypertension, Diabetes Mellitus, Congestive heart failure, Valvular heart disease, Peripheral vascular disease, Chronic lung disease, Obesity, Peptic ulcer disease, Coagulopathy, Liver disease, Solid tumor, deficiency anemia, Sarcoidosis, , Dementia, History of myocardial infarction, history of percutaneous coronary intervention (PCI) and history of coronary artery bypass graft (CABG). Logistic regression was used for dichotomous outcomes 
and linear regression was used for continuous outcomes. All P-values were 2-sided, with 0.05 as threshold for statistical significance.

ICD 10 codes used in our analysis are included in supplementary table 1

\section{Results:}

\section{Patient characteristics}

From 107 million discharges included in NRD 2016-2018, we identified a total of 7212 patients who presented with ventricular tachycardia and had catheter ablation. Baseline characteristics were stratified according to presence or absence of chronic kidney disease. 1679 patients had CKD and 5533 did not.

Mean age was higher in CKD group with $67.9( \pm 11.09)$ and $60.5( \pm 14.56)$ in non-CKD group. Female sex constituted $12 \%$ in patients with CKD and $21 \%$ in those without. CKD group was associated with Charlson comorbidity index score of 3 or more ( $98 \%$ vs $41 \%$ ), congestive heart failure (92\% vs $71 \%$ ), history of MI ( $40 \%$ vs $32 \%$ ), history of PCI ( $28 \%$ vs $21 \%$ ), history of CABG ( $31 \%$ vs $18 \%$ ), diabetes mellitus ( $46 \%$ vs $26 \%$ ), and dyslipidemia (56\% vs $49 \%$ ). Other patient and hospital characteristics were not different between the two groups (Table 1).

\section{Outcomes}

From a total cohort of 7212 hospitalizations with a primary diagnosis of ventricular and underwent catheter ablation, a total of $232(3.2 \%)$ patients had in-hospital mortality; 113 patients had CKD and 119 did not have CKD. CKD was associated with increased odds of mortality. Adjusted OR was 2.24, 95\% CI (1.29-3.88), $\mathrm{p}<0.01$.

From 292 readmissions carried a primary diagnosis of ventricular tachycardia, 88 of which were patients with CKD on index admission. CKD on index admission was not associated with increased risk of 30-day readmission due to ventricular tachycardia. Adjusted OR was 1.35, 95\% CI (0.78-2.31), P=0.27.

In terms of acute kidney injury 1348 had acute kidney injury of whom 726 patients had baseline CKD. Presence of CKD was associated with increased risk of acute kidney injury in adjusted multivariate regression model. Adjusted OR was 3.69 95\% CI (2.87-4.74), $\mathrm{P}<0.01$.

Mean length of stay in our cohort was 6.40 days. Mean length of stay in patients without and with CKD was 5.69 and 8.73 days, respectively. After adjustment, patients with CKD had increased mean length of stay of 1.58 days and the difference was statistically significant, $\mathrm{P}<0.01$. (Table 2 )

Mean hospital charges in our cohort was 188014 USD. Mean hospital charges in patients without and with CKD was 176249 USD and 226796 USD, respectively. After adjustment, there was a mean difference of 27599 USD and the difference was statistically significant, $\mathrm{P}=0.012$. (Table 2)

\section{Subgroup analysis:}

Subgroup analysis was done based on CKD stages; III, IV, V, and ESRD. CKD stages I and II were excluded from subgroup analysis due to low number of observations. CKD stage IV was associated with increased odds of mortality in adjusted multivariate regression model. Adjusted OR was 4.48 95\% CI 1.79 - 11.2, $\mathrm{P}<0.01$. CKD stages III, IV, and V were associated with increased odds of acute kidney injury with adjusted multivariate regression model Adjusted OR were 3.7 (95\% CI $2.7-5.1, \mathrm{P}<0.01$ ), 5.5 (95\% CI 3.1 - 9.7, $\mathrm{P}<0.01$ ), 25.7 (95\% CI $2.6-246, \mathrm{P}=0.005$ ), respectively. (Table 3)

CKD stages III, IV, V and ESRD were associated with statistically significant increase in mean length of stay by $1.37(\mathrm{P}<0.01), 2.99(\mathrm{P}<0.01), 19.27(\mathrm{P}<0.01)$ and $6.01(\mathrm{P}<0.01)$ days, respectively. CKD stage $\mathrm{V}$ and ESRD were associated with statistically significant increase in mean total hospital charges by 343797 USD $(\mathrm{P}=0.020)$ and 150610 USD $(\mathrm{P}=0.040)$, respectively. (Table 3$)$.

\section{Discussion:}


Over the last decade, the utilization rate of catheter ablation (CA) has steadily increased as an important therapy for ventricular tachycardia (VT). [7] Despite significant advancements in electroanatomic mapping systems and imaging technologies, VT ablation is still associated with a risk of significant complications. A decision to perform the procedure includes balancing those risks against potential benefits. [8] Typically, patients with VT have complex underlying comorbidities that contribute to morbidity and mortality following the ablation procedure. [9] Previous clinical trials have been conducted to assess the efficacy and safety of VT ablation. [10] However, many of these studies have excluded specific populations, such as those with renal dysfunction. [11] Our study aims to fill this gap by addressing the association of chronic kidney disease (CKD) with in-hospital mortality and complications following VT ablation using a real-world large administrative database. Proper understanding of the risks of VT ablation in this population will have significant clinical implications on CKD patients with VT, by identifying therapeutic strategies that can improve survival and by guiding patient counseling.

In-hospital mortality has been widely used in the literature as an important outcome measure for invasive cardiology procedures. In this multi-center registry, in-hospital mortality was our primary outcome, and it occurred in $3.2 \%$ of VT patients undergoing CA, higher than the mortality rates reported in previous similar studies. A retrospective study from National Inpatient Sample (NIS) database (2006-2013), have revealed a $2.8 \%$ in-hospital mortality rate among 25,451 patients undergoing VT ablation. [5] The higher mortality rate in recent years could be due to the increased number of patients with a higher burden of comorbidities undergoing VT ablation or due to a rising number of low-volume centers performing VT ablation with decreased access to equipment and personnel. [7] Our study adds to the current literature by highlighting the association between in-hospital mortality and CKD status $(\mathrm{OR}=2.24 ; 95 \%$ CI: $1.29-3.88, \mathrm{p}=0.004)$, particularly CKD stage IV $(\mathrm{OR}=4.4895 \%$ CI $1.79-11.2, \mathrm{p}<0.01)$. These findings are comparable with Palanismawy et al. who reported a similar association between CKD and in-hospital mortality following CA $(\mathrm{OR}=2.15 ; 95 \%$ CI: 1.12-4.14, $\mathrm{p}=0.022)$, among 81,539 patients with postinfarct VT identified through NIS. $[7]$

Thirty-day readmission is another important indicator for the success of the VT ablation procedure in highrisk patients. [12] According to Sharma et al, who used NRD data from 2010 to 2014, 1 in 6 patients was readmitted within 30 days after undergoing VT ablation. The most common cause of 30-day readmission after the index hospitalization for VT ablation was ventricular arrhythmia (39.51\%) followed by acute congestive heart failure (11.83\%). Moreover, they investigated the different predictors of 30-day readmission and found a significant association with CKD ( $\mathrm{OR}=1.3 ; 95 \%$ CI: 1.1-1.6, $\mathrm{p}=0.01)$. [3] Our study using the same database from 2016 to 2018 did not find a statistically significant association between CKD status and 30 -day readmission rates due to ventricular arrhythmia after adjusting for confounders $(\mathrm{OR}=1.35 ; 95 \%$ CI: $0.78-2.31, \mathrm{p}=0.27)$.

Given the complexity of VT catheter ablation procedure and the high comorbidity burden among patients undergoing this procedure, the length of stay and the hospital costs hospitalizations following the procedure are significant. [13] A study conducted by Cheung et al reported a median length of stay of the index admission of 4.3 days, while the mean length of stay in our study was 6.4 days (8.73 in CKD patients). [12] Moreover, we found that the mean hospital charges in CKD patients undergoing VT ablation were 226,796 USD, compared with 176,249 USD in patients without CKD. These associations were most significant in patients with CKD stage V. This remarkable difference in length of stay and hospital costs could have important implications on resource allocation in hospitals performing VT ablation.

The association between CKD and worse outcomes in patients undergoing VT ablation is complex and could be related to structural cardiac abnormalities caused by CKD, in addition to several triggers in this population. Firstly, CKD leads to progressive coronary calcification and coronary artery disease, which is considered the most common etiology of VT in these patients also CKD can make intra-op volume management more challenging, thus CKD may be related to less complete ablation. [14] CKD patients are also at risk for QTc-prolongation due to impairment of cardiac repolarization mechanisms. [15] In addition, CKD patients undergoing hemodialysis are affected by electrolyte shifts, such as sudden potassium and calcium 
shifts, leading to a mechanical and electrical imbalance in the cardiac myocytes. [16] Other underlying mechanisms for this imbalance could be attributed to oxidative stress, increased levels, and the accumulation of several cardiotoxic substances such as homocysteine b2-microglobulin, and parathyroid hormone [17] The present study contributes to a better understanding of this high-risk subgroup of patients undergoing VT ablation. Development of a nationally valid risk stratification model and improving post-hospital care have the potential to offset some of the risks inherent to ablating VT in CKD patients.

\section{Limitations}

Studies based on an administrative database, such as the NRD, have inherent limitations. Firstly, there is a risk of error secondary to variations in coding practices. Additionally, data solely related to 30-day readmissions after an index hospitalization is the available information pertaining to the longitudinal followup of patients, information related to race and ethnicity, and individual operator and procedure level are not available in the NRD. Lastly, the NRD does not include out-of-hospital deaths or emergency room mortality. Other factors that can affect a patient's prognosis, like drugs are not included in the database as well. The large sample size included in our study can partially offset some of these limitations, and the real-world clinical experience can add to the currently existing literature.

\section{Conclusion}

VT ablation in patients with CKD results in increased incidence of adverse clinical events such as greater in-hospital mortality and acute kidney injury, in addition to increased length of stay and higher hospital charges. CKD IV and III had higher hospital mortality and 30-day readmission rate, respectively compared with other CKD stages. Understanding risk of VT ablation in patients with different stages of CKD will inform discussions of risks and benefits of of patients with CKD being considered for catheter ablation of VT.

\section{References}

1. Kuriachan, V.P., G.L. Sumner, and L.B. Mitchell, Sudden Cardiac Death. Current Problems in Cardiology, 2015. 40 (4): p. 133-200.

2. de Bakker, J.M., et al., Slow conduction in the infarcted human heart. 'Zigzag' course of activation. Circulation, 1993.88 (3): p. 915-26.

3. Sharma, P., et al., Short-term outcomes associated with inpatient ventricular tachycardia catheter ablation. Pacing Clin Electrophysiol, 2020. 43 (5): p. 444-455.

4. Maskoun, W., et al., Outcome of catheter ablation for ventricular tachycardia in patients with ischemic cardiomyopathy: A systematic review and meta-analysis of randomized clinical trials. Int J Cardiol, 2018. 267 : p. $107-113$.

5. Gunda, S., et al., Risk score model for predicting complications in patients undergoing ventricular tachycardia ablation: insights from the National Inpatient Sample database. Europace, 2019.21 (3): p. 475-483.

6. Bloom JE, Dinh DT, Noaman S, et al. Adverse impact of chronic kidney disease on clinical outcomes following percutaneous coronary intervention. Catheter Cardiovasc Interv. 2020;1-9.https://doi.org/10.1002/ccd. 29436).

7. Palaniswamy, C., et al., Catheter ablation of postinfarction ventricular tachycardia: ten-year trends in utilization, in-hospital complications, and in-hospital mortality in the United States. Heart Rhythm, 2014. 11 (11): p. 2056-63.

8. Campbell, T., et al., Updates in Ventricular Tachycardia Ablation. Korean Circ J, 2021. 51 (1): p. 15-42.

9. Cronin, E.M., et al., 2019 HRS/EHRA/APHRS/LAHRS expert consensus statement on catheter ablation of ventricular arrhythmias.Europace, 2019. 21 (8): p. 1143-1144. 
10. Pothineni, N.V., et al., Complication rates of ventricular tachycardia ablation: Comparison of safety outcomes derived from administrative databases and clinical trials. Int J Cardiol, 2015.201 : p. 529-31.

11. Maini, R., et al., Persistent Underrepresentation of Kidney Disease in Randomized, Controlled Trials of Cardiovascular Disease in the Contemporary Era. J Am Soc Nephrol, 2018. 29 (12): p. 2782-2786.

12. Cheung, J.W., et al., Outcomes, Costs, and 30-Day Readmissions After Catheter Ablation of Myocardial Infarct-Associated Ventricular Tachycardia in the Real World: Nationwide Readmissions Database 2010 to 2015. Circ Arrhythm Electrophysiol, 2018. 11 (11): p. e006754.

13. Calkins, H., et al., Cost-effectiveness of catheter ablation in patients with ventricular tachycardia. Circulation, 2000.101 (3): p. 280-8.

14. Stompor, T., Coronary artery calcification in chronic kidney disease: An update. World J Cardiol, 2014. 6 (4): p. 115-29.

15. Liu, P., et al., Acquired long QT syndrome in chronic kidney disease patients. Ren Fail, 2020. 42 (1): p. 54-65.

16. Bonato, F.O.B. and M.E.F. Canziani, Ventricular arrhythmia in chronic kidney disease patients. J Bras Nefrol, 2017. 39 (2): p. 186-195.

17. Karmin O, Siow YL. Metabolic imbalance of homocysteine and hydrogen sulfide in kidney disease. Curr Med Chem. 2018;25(3):367-377..

\section{Figure Legends:}

Figure 1: baseline characteristics of study population

Figure 2: Forest Plot of main study outcomes.

Figure 3: central illustration of the study

Table 1: baseline characteristics of the study population

\begin{tabular}{llll}
\hline Characteristics & Chronic kidney disease & Chronic kidney disease & P value \\
\hline & Absent $(\mathbf{n = 5 5 3 3})$ & Present $(\mathbf{n = 1 6 7 9})$ & \\
Female & $1,185(21 \%)$ & $205(12 \%)$ & $<0.01$ \\
Mean Age in years (SD) & $60.5( \pm 0.7)$ & $67.9( \pm 0.8)$ & $<0.01$ \\
Age category & & & \\
18-45 & $738(13 \%)$ & $39(2 \%)$ & \\
$45-65$ & $2,297(42 \%)$ & $527(31 \%)$ & \\
$65-90$ & $2,485(45 \%)$ & $1,100(66 \%)$ & \\
$>90$ & $13(<1 \%)$ & $13(<1 \%)$ & \\
Smoking & $70(1 \%)$ & $24(1 \%)$ & \\
Charlson comorbidities index score & & & \\
0 & $836(15 \%)$ & $<11$ & \\
1 & $976(18 \%)$ & $<11$ \\
2 & $1,439(26 \%)$ & $31(2 \%)$ & \\
3 & $2,282(41 \%)$ & $1,648(98 \%)$ & \\
Median household income quartile for zip code & & & \\
0-25th percentile & $1,261(23 \%)$ & $463(28 \%)$ & \\
26th to 50th percentile & $1,478(27 \%)$ & $474(29 \%)$ & \\
51st to 75th percentile & $1,442(26 \%)$ & $364(22 \%)$ & \\
76th to 100th percentile & $1,287(24 \%)$ & $359(22 \%)$ & \\
Insurance & & & \\
Medicare & $2788(50 \%)$ & $1233(74 \%)$ &
\end{tabular}




\begin{tabular}{llll}
\hline Characteristics & Chronic kidney disease & Chronic kidney disease & P value \\
\hline Medicaid & $468(8 \%)$ & $123(7 \%)$ & \\
Private & $1,964(36 \%)$ & $257(14 \%)$ & \\
Self-pay & $101(2 \%)$ & $13(1 \%)$ & \\
Hospital bed size & & & \\
Small & $207(4 \%)$ & $64(4 \%)$ & \\
Medium & $1,101(20 \%)$ & $294(17 \%)$ & \\
Large & $4,225(76 \%)$ & $1,321(79 \%)$ & \\
Teaching hospital & $4,964(90 \%)$ & $1,530(91 \%)$ & 0.22 \\
Comorbidities & & & \\
Hypertension & $3,722(67 \%)$ & $1,562(93 \%)$ & $<0.01$ \\
History of MI & $1,766(32 \%)$ & $675(40 \%)$ & $<0.01$ \\
History of PCI & $1,179(21 \%)$ & $475(28 \%)$ & $<0.01$ \\
History of CABG & $1,013(18 \%)$ & $527(31 \%)$ & $<0.01$ \\
Congestive heart failure & $3,955(71 \%)$ & $1,538(92 \%)$ & $<0.01$ \\
Valvular heart disease & $786(14 \%)$ & $320(19 \%)$ & 0.004 \\
Peripheral vascular disease & $2,908(53 \%)$ & $1,175(70 \%)$ & $<0.01$ \\
Chronic lung disease & $1,046(19 \%)$ & $435(26 \%)$ & $<0.01$ \\
obesity & $1,010(18 \%)$ & $312(19 \%)$ & 0.84 \\
dementia & $34(1 \%)$ & $27(2 \%)$ & 0.008 \\
Hypothyroidism & $687(12 \%)$ & $361(21 \%)$ & $<0.01$ \\
Coagulopathy & $286(5 \%)$ & $165(10 \%)$ & $<0.01$ \\
Solid tumor without metastasis & $53(1 \%)$ & $27(2 \%)$ & 0.104 \\
Deficiency anemia & $105(2 \%)$ & $67(4 \%)$ & 0.001 \\
Dyslipidemia & $2,695(49 \%)$ & $942(56 \%)$ & 0.0005 \\
Diabetes & $1,454(26 \%)$ & $774(46 \%)$ & $<0.01$ \\
Liver disease & $163(3 \%)$ & $87(5 \%)$ & 0.001 \\
Sarcoidosis & $127(21 \%)$ & $25(1 \%)$ & 0.14 \\
\hline
\end{tabular}

+MI: myocardial infarction, ++PCI: percutaneous coronary intervention, §CABG: coronary artery bypass graft

\section{Table 2: study outcomes}

\begin{tabular}{llll}
\hline Outcome & Chronic kidney disease & Chronic kidney disease & P value \\
\hline & Absent $(\mathbf{n = 5 5 3 3})$ & Present $\mathbf{( n = 1 6 7 9 )}$ & \\
In-hospital mortality (n) & 119 & 113 & $<0.01$ \\
Adjusted odds ratio & Reference & $2.24,95 \%$ CI $(1.29-3.88)$ & $<0.01$ \\
30-day readmission due to Ventricular tachycardia (n) & 204 & 88 & $<0.01$ \\
Adjusted odds ratio & & $1.35,95 \%$ CI $(0.78-2.31)$ & 0.27 \\
Stroke and TIA (n) & 14 & $<11$ & 0.25 \\
Adjusted odds ratio & Reference & $0.6595 \%$ CI $(0.13-3.1)$ & 0.59 \\
Acute kidney injury (n) & 623 & 726 & $<0.01$ \\
Adjusted odds ratio & Reference & $3.6995 \%$ CI $(2.87-4.74)$ & $<0.01$ \\
Access site bleeding (n) & 66 & 31 & 0.09 \\
Adjusted odds ratio & Reference & $0.9295 \%$ CI $(0.46-1.85)$ & 0.82 \\
Iatrogenic cardiac complication (n) & 59 & 13 & 0.5 \\
Adjusted odds ratio & Reference & $0.695 \%$ CI $(0.16-2.24)$ & 0.45 \\
Acute myocardial infarction (n) & 183 & 82 & 0.019 \\
Adjusted odds ratio & Reference & $1.0295 \%$ CI $(0.64-1.63)$ & 0.91
\end{tabular}




\begin{tabular}{llll}
\hline Outcome & Chronic kidney disease & Chronic kidney disease & P value \\
\hline Hemopericardium (n) & 21 & $<11$ & 0.83 \\
Adjusted odds ratio & Reference & $1.1495 \%$ CI $(0.17-7.3)$ & 0.88 \\
Mean length of stay (days) & 5.69 & 8.72 & $<0.01$ \\
Adjusted mean difference (days) & & 1.58 & $<0.01$ \\
Mean total charges (USD) & 176,249 & 226,796 & $<0.01$ \\
Adjusted mean total charges difference (USD) & & 27,599 & 0.012 \\
\hline
\end{tabular}

$+\mathrm{n}$ : number, ++ TIA: transient ischemic attack, $\S$ CI: confidence interval

Table 3: subgroup analysis according to CKD stage and outcomes

\begin{tabular}{lllll}
\hline outcome & CKD III & P value & CKD IV & P v \\
\hline In-hospital mortality & 50 & & 15 & \\
Adjusted odds ratio & $1.9995 \%$ CI $0.99-4.01$ & 0.052 & $4.4895 \%$ CI $1.79-11.2$ & 0.00 \\
30-day readmission due to Ventricular tachycardia (n) & 53 & & $<11$ & \\
Adjusted odds ratio & $1.895 \%$ CI $0.9-3.6$ & 0.08 & $0.7495 \%$ CI $0.16-3.3$ & 0.7 \\
Acute kidney injury (n) & 338 & & 72 & $<$. \\
Adjusted odds ratio & $3.795 \%$ CI $2.7-5.1$ & $<0.01$ & $5.595 \%$ CI $3.1-9.7$ & $<.91$ \\
Mean LOS (days) & 8.75 & & 2.99 & $<0$. \\
Adjusted mean LOS difference (days) & 1.37 & & 227267 & \\
95\% CI & $0.34-2.39$ & & 34972 & \\
Mean Hospital charges (USD) & 218014 & 0.545 & $-17562-87507$ & \\
Adjusted mean hospital charges difference (USD) & 6588 & & & 0.19 \\
95\% CI & $-14738-27914$ & & \\
\hline
\end{tabular}

+CKD: chronic kidney disease, ++ n: number, § ESRD: end stage renal disease, I n: number, \# CI: confidence interval

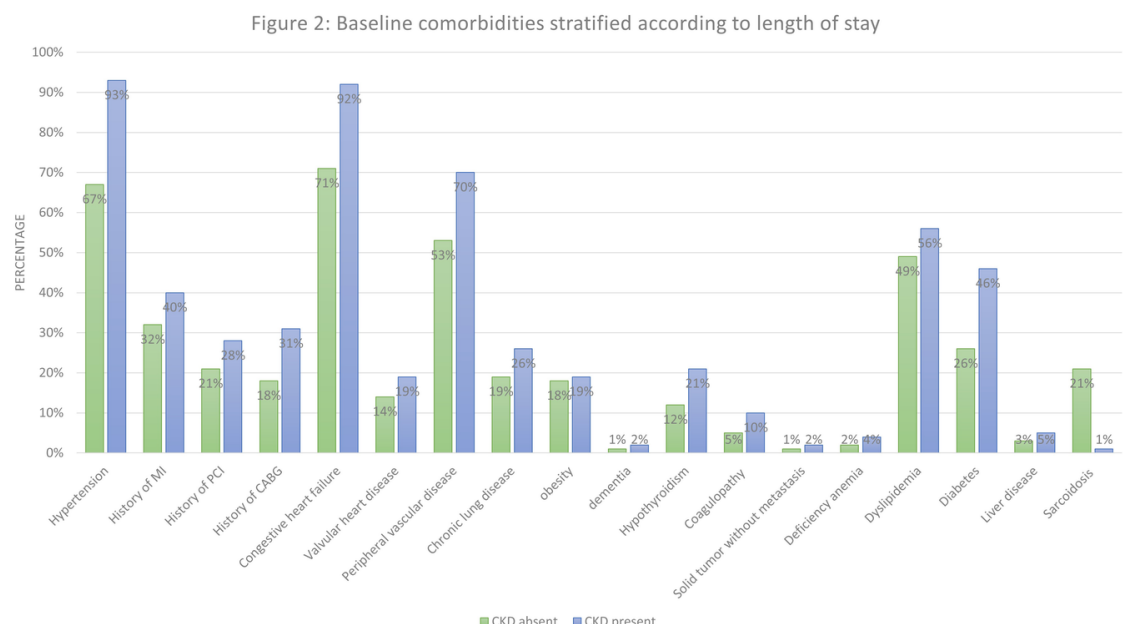



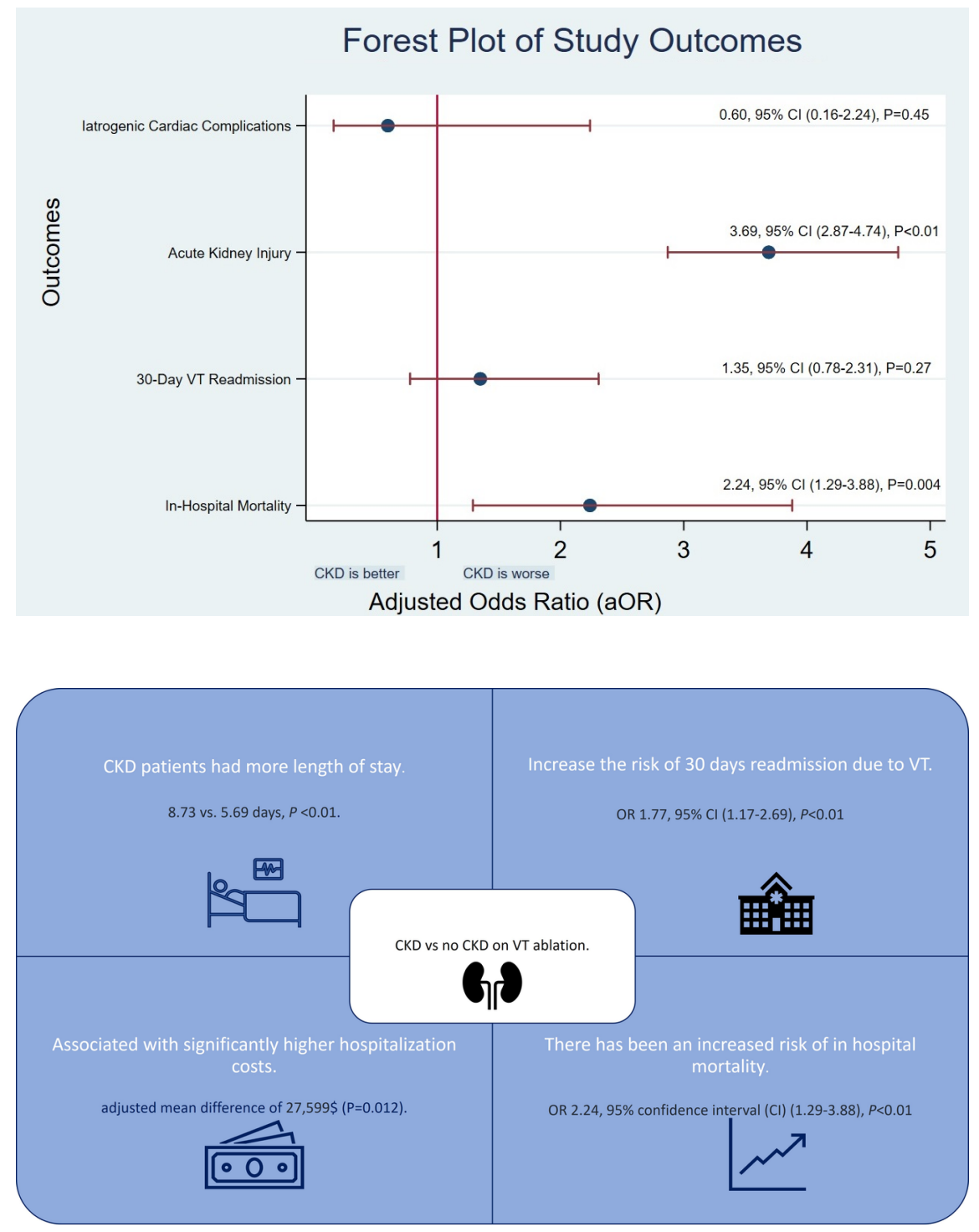